\title{
Supplementation with low concentrations of melatonin improves nuclear maturation of human oocytes in vitro
}

\author{
Duo Wei • Culian Zhang • Juanke Xie $\cdot$ Xiaobing Song • \\ Baoli Yin • Qi Liu • Lin Hu • Haoying Hao • \\ Jiaxuan Geng $\cdot$ Peng Wang
}

Received: 9 February 2013 / Accepted: 24 May 2013 / Published online: 6 June 2013

(C) Springer Science+Business Media New York 2013

\begin{abstract}
Purpose Studies in bovine and porcine have indicated that melatonin (MT) could induce meiotic maturation of immature oocytes in vitro. The object of the current study was to investigate if MT could ameliorate human oocytes maturation during rescue in vitro maturation (IVM).

Methods Two hundred seventy eight germinal vesicle (GV) oocytes and 451 (MI) metaphase I oocytes were vitrified, thawed and then matured in vitro. All the oocytes were randomly allocated into six groups in which the oocytes were cultured in medium supplemented with different concentrations of MT $\left(0,10^{-2}, 1,10^{2}, 10^{4}, 10^{6} \mathrm{nM}\right)$ and nuclear maturation was evaluated at $6 \mathrm{~h}, 12 \mathrm{~h}, 18 \mathrm{~h}, 24 \mathrm{~h}$ and $48 \mathrm{~h}$ of culture.

Results The optimal MT concentration for both GV and MI oocytes was $1 \mathrm{nM}$. At $24 \mathrm{~h}$ of culture, nuclear maturation rate of MI oocytes cultured in $1 \mathrm{nM}$ MT medium was significantly higher than other groups $(P<0.05)$; Nuclear maturation rate of GV oocytes cultured in $1 \mathrm{nM}$ MT medium was also
\end{abstract}

Funding information The current study is funded by Major Program of Key Medical Science Project of Henan Province (Funding Number:201001010).

Capsule Low concentration of exogenous melatonin could ameliorate nuclear maturation of human oocyte during rescue IVM, while high concentration of melatonin presented negative effects.

D. Wei $\cdot$ C. Zhang $(\bowtie) \cdot J$. Xie $\cdot$ X. Song $\cdot$ B. Yin $\cdot$ Q. Liu

L. Hu $\cdot$ H. Hao $\cdot$ J. Geng

Reproductive Medicine Center, the People's Hospital of

Zhengzhou University; Henan Provincial People's Hospital,

Zhengzhou, Henan, China

e-mail: luckyzcl@yahoo.com.cn

P. Wang

Center for Reproductive Medicine, Provincial Hospital Affiliated

to Shandong University, Jinan, China significantly higher than the control group $(P<0.05)$. On the other hand, decreased nuclear maturation rate was observed in the high MT concentration group $\left(10^{6} \mathrm{nM}\right)$.

Conclusions The current study demonstrated that low concentration of exogenous MT could ameliorate nuclear maturation of human oocyte during rescue IVM, while high concentration of MT presented negative effects.

Keywords Melatonin $\cdot$ Human immature oocyte $\cdot$ In vitro maturation $\cdot$ Vitrification

\section{Introduction}

Early in 1965, Robert G. Edwards discovered that morphologically immature human oocytes could spontaneously mature in vitro and be subsequently fertilized [5]. Eighteen years later, the first in vitro maturation (IVM) baby came into this world [30]. It is estimated that there had been over 1300 IVM babies delivered worldwide till 2009 [6]. Nowadays, because of the well acknowledged benefits of this technique, IVM has emerged as an important and prosperous field of assisted reproductive technique (ART). IVM is safe and costeffective especially when it is applied to women with polycystic ovary syndrome (PCOS) [3]. Occasionally, it could also be applied to poor responders and cancer patients [18].

Compared to conventional ovarian superstimulation cycles, the yield of IVM cycles is relatively unsatisfactory. And so far, there are no universal IVM protocols. In order to achieve better results, various modifications (e.g. hormone priming, cycle monitoring and culture conditions) have been tried [15]. When it comes to in vitro culture conditions, oxidative stress is one of the most devastating factors [4]. In vivo, oocytes and embryos produce endogenous reactive oxygen species (ROS) [9]. At the same time, damaging effect of 
ROS could be balanced by the antioxidant defense system in vivo, which consists of super oxide dismutase (SOD) and glutathione (GSH). Particularly, antioxidant has been found in follicles and fluid of fallopian tubes [12]. In vitro, a variety of factors (e.g. visible light, elevated oxygen concentrations, and unusual concentrations of metabolites and substrates) could also result in unnecessary exposure to ROS [7]. Because of a lack of antioxidant system and the extra exogenous ROS, it is reasonable to employ medium supplemented with biologically active antioxidant compounds to balance endogenous and exogenous ROS in vivo.

Melatonin (MT), which is secreted mainly by pineal gland, is in charge of mediating a number of circadian and seasonal processes in mammalian reproduction [16, 19]. MT and its metabolites have been demonstrated to be powerful and direct scavenger of free radicals [20, $21,27,28]$. In contrast to other known radical scavengers, the function of MT is multi-faceted and universal $[8,13,29]$. The beneficial effects of MT have been reported in rodent and bovine in vitro embryo development $[10,17]$. And according to toxicity test of MT, no detrimental effects were found on rodent embryo development [2, 11, 14]. MT is effective, relatively safe and hence promising as supplement of IVM medium. But there has been no research exploring the effects of MT in human oocyte in vitro development so far. We initiated this study to examine if MT could ameliorate meiotic maturation of human oocytes in vitro. Because this is a pilot study and this is also the first application on human oocytes, we used the immature oocytes from superovulation cycles, instead of immature oocytes from typical IVM cycles.

\section{Material and methods}

Patients

In this study, we enrolled 438 women undergoing intracytoplasmic sperm injection (ICSI) cycles at the center for reproductive medicine of Henan Provincial Hospital from May 2011 to July 2012, according to the following criteria: 1) younger than 35 years old; 2) patients undergoing their first ICSI cycles; 3) cycles stimulated with standard long pituitary down-regulation protocol; 4); non-smoking and non-drinking. We have obtained ethics approval from institutional review board of Henan Provincial Hospital that, after obtaining informed consents from the involved patients, their immature oocytes could be used for scientific research.

Ovarian superstimulation and oocyte retrieval

All the ICSI cycles were carried out according to the standard protocol of our center which was described elsewhere

Fig. 1 Enrollment and grouping

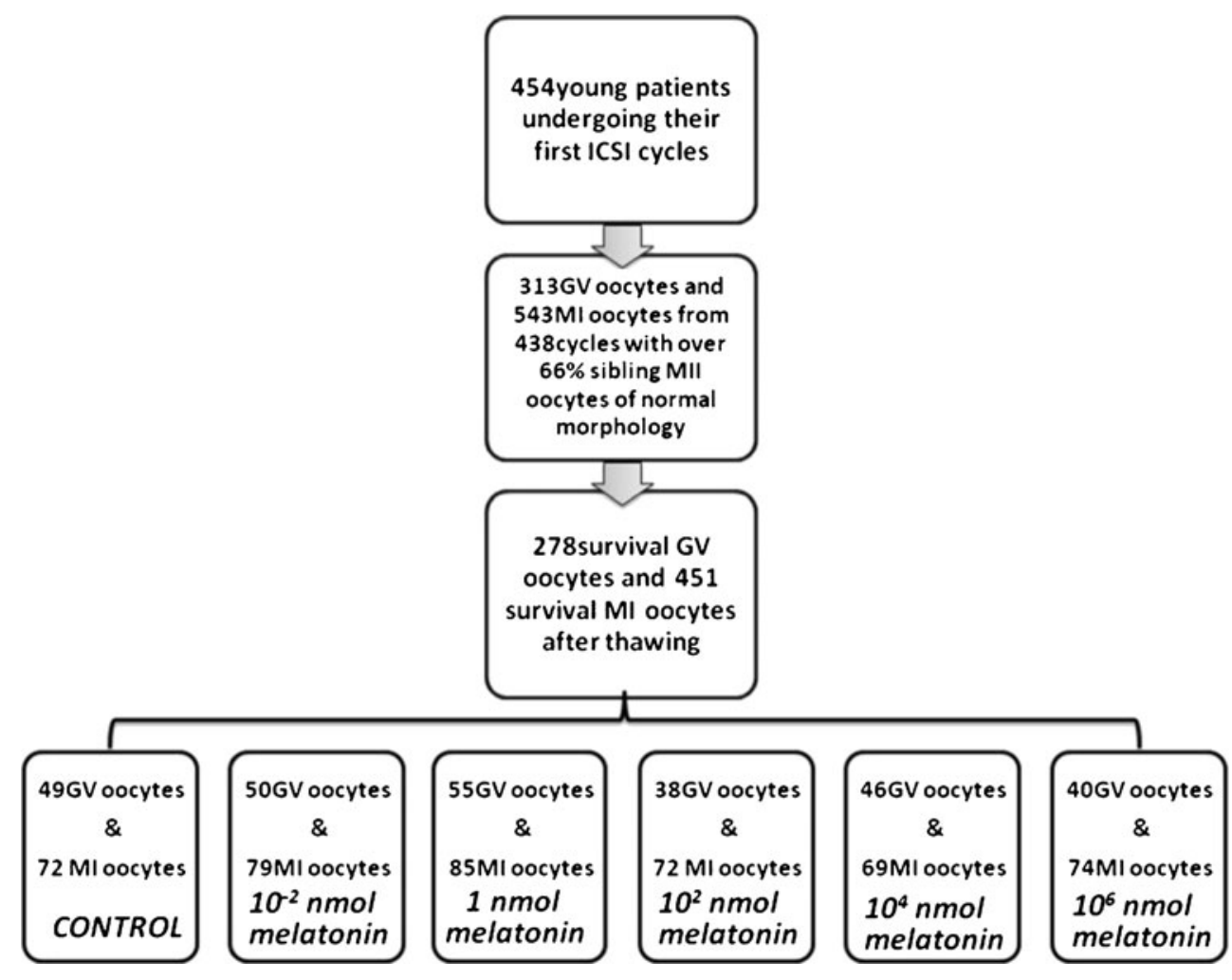


[31]. After pituitary down-regulation by gonadotropin releasing hormone agonist (GnRH-agonist) (Triptorelin Acetate, Ferring AG, Switzerland), ovarian stimulation was initiated by recombinant FSH (Gonal-F; Serono, Switzerland). When 3 or more follicles reached $18 \mathrm{~mm}$ in diameter, 6,000 10,000 IU human chorionic gonadotropin (hCG) (Chorionic Gonadotrophin of human; Lizhu, China) was administered. After $36 \mathrm{~h}$, oocyte cumulus complexes (OCC) were retrieved. Removal of the surrounding cumulus cells was accomplished by a combined enzymatic and mechanical treatment under a stereoscopic dissecting microscope. Mature oocytes were fertilized and further cultured, whilst germinal vesicle breakdown/metaphase I (MI) and germinal vesicle (GV) oocytes were vitrified (Kitizato, Japan). In order to avoid intrinsic abnormalities of the oocytes, cycles with more than $33 \%$ sibling immature oocytes were excluded in this study. Totally, $313 \mathrm{GV}$ oocytes and $543 \mathrm{MI}$ oocytes were vitrified and stored in liquid nitrogen.

\section{Rescue IVM}

Two hundred seventy eight GV oocytes and 451 MI oocytes survived after thawing. The oocytes were then randomly transferred into the medium supplemented with different concentrations of MT (Sigma-Aldrich) $\left(10^{-2}, 1,10^{2}, 10^{4}\right.$ and $\left.10^{6} \mathrm{nM}\right)$ and the control medium. The basic maturation media consisted of commercial SAGE IVM media (SAGE, USA), 75 mIU FSH (Ferring, Germany) and 75 mIU LH (Ferring, Germany). Each oocyte was cultured in a $50 \mu \mathrm{L}$ microdrop for $48 \mathrm{~h}$ in an atmosphere of $5 \% \mathrm{O}^{2}, 5 \% \mathrm{CO}^{2}$ and $95 \%$ relative humidity at $37{ }^{\circ} \mathrm{C}$.

Assessment of meiotic status

The oocytes were observed by one embryologist after $6 \mathrm{~h}$, $12 \mathrm{~h}, 18 \mathrm{~h}, 24 \mathrm{~h}$ and $48 \mathrm{~h}$ of culture. Oocytes that displayed PB1 were considered as nuclear maturation (MII). The oocytes with an intra cytoplasmatic nucleus were identified as GV, while the oocytes that had neither a visible GV nor PB1 were classified as MI.

\section{Statistical analysis}

For independent samples, student's $t$-test was employed to analyze normally distributed quantitative variables, while the chi-square test or Fisher's test was used to analyze nominal variables. Continuous variables were summarized with descriptive statistics ( $n$, mean, standard deviation). Categorical variables were described with counts and percentages. All tests were two-sided, and a $P$-value less than 0.05 are considered statistically significant for all statistical tests. All analyses were performed using SPSS software, version 18.

\section{Results}

Epidemiologic and clinical characteristics of the subjects

At first we enrolled 454 young patients undergoing their first ICSI cycle. 16 cycles were excluded because of meiosis retardation or poor oocyte morphology. Totally,313 GV oocytes and 543 MI oocytes were vitrified. After freezing and thawing, $278 \mathrm{GV}$ oocytes and 451 MI oocytes survived, and they were randomly assigned into the control group and five other groups of varying MT concentrations (Fig. 1). No significant differences were found in major clinical characteristics among the six groups (Table 1).

Low concentrations of MT could accelerate nuclear maturation

Effects of MT on nuclear maturation of GV and MI oocytes are shown in Fig. 2 respectively.

For the GV oocytes, during the first $6 \mathrm{~h}$ of culture, none of the oocytes presented meiosis related morphological change. During the second $6 \mathrm{~h}(6-12 \mathrm{~h}$ of culture), a small portion of

Table 1 Epidemiologic and clinical characteristics of controls and 5 groups supplemented with different concentrations of MT

\begin{tabular}{|c|c|c|c|c|c|c|}
\hline $\begin{array}{l}\text { MT concentration } \\
(\mathrm{nM})\end{array}$ & $\begin{array}{l}\text { Number of oocytes/ } \\
\text { patients }\end{array}$ & Age (years) & BMI & $\begin{array}{l}\text { Basal FSH (IU/ } \\
\mathrm{ml})\end{array}$ & $\begin{array}{l}\text { Total Gn dose } \\
\text { (IU) }\end{array}$ & $\begin{array}{l}\text { Immature oocyte rate } \\
(\%)\end{array}$ \\
\hline Control & $121 / 73$ & $28.55 \pm 4.63$ & $23.68 \pm 3.73$ & $7.34 \pm 2.82$ & $2037.05 \pm 879.23$ & $86.5 \pm 5.82$ \\
\hline $10^{-2}$ & $129 / 73$ & $28.12 \pm 5.20$ & $23.41 \pm 3.24$ & $7.45 \pm 2.20$ & $2090.25 \pm 902.33$ & $86.7 \pm 5.88$ \\
\hline 1 & $140 / 73$ & $29.22 \pm 4.45$ & $22.23 \pm 2.95$ & $7.50 \pm 2.58$ & $1938.78 \pm 850.65$ & $85.6 \pm 6.90$ \\
\hline $10^{2}$ & $110 / 73$ & $29.45 \pm 4.61$ & $22.53 \pm 3.74$ & $7.65 \pm 2.49$ & $2075.45 \pm 912.36$ & $85.0 \pm 6.39$ \\
\hline $10^{5}$ & $115 / 73$ & $28.59 \pm 5.59$ & $22.25 \pm 3.08$ & $8.02 \pm 2.86$ & $2020.53 \pm 898.41$ & $85.0 \pm 6.16$ \\
\hline $10^{7}$ & $114 / 73$ & $28.89 \pm 4.63$ & $23.58 \pm 3.12$ & $8.06 \pm 2.68$ & $1998.25 \pm 890.02$ & $84.7 \pm 7.07$ \\
\hline
\end{tabular}


the GV oocytes cultured in the media supplemented with low concentrations of MT $\left(10^{-2} \mathrm{nM}, 1 \mathrm{nM}\right.$ and $10^{2} \mathrm{nM}$ ) (low-MT groups) successfully proceeded to the MII stage, while all the GV oocytes of the control group and those cultured in medium supplemented with high concentrations of MT $\left(10^{4} \mathrm{nM}\right.$ and $\left.10^{6} \mathrm{nM}\right)$ (high-MT groups) remained to be nucleic immature. During the third $6 \mathrm{~h}$ (12-18 $\mathrm{h}$ of culture), the GV oocytes of the control group and high-MT groups began to present the first polar body (1 $\mathrm{PB})$, however, growth of nuclear maturation rate was still slower than that of the low-MT groups. During the fourth $6 \mathrm{~h}$, growth of nuclear maturation rate of the high-MT group remained to be the slowest. Of note, statistically significant increase of nuclear maturation rate was observed during the second $6 \mathrm{~h}$ and the third $6 \mathrm{~h}$ in the $1 \mathrm{nM}$ MT group and the $10^{2} \mathrm{nM}$ MT group respectively.

Similar phenomenon was observed in the MI oocytes, faster growth of nuclear maturation rate was observed in the
low-MT groups. Notably, statistically significant increase of nuclear maturation rate was observed during the 1 st and 2 nd $6 \mathrm{~h}$ in both the $1 \mathrm{nM}$ and $10^{-2} \mathrm{nM}$ MT groups.

Low concentration of MT could increase nuclear maturation rate

For the GV oocytes, at $24 \mathrm{~h}$ of culture, nuclear maturation rate of the low-MT groups were higher than the control group. And the difference of nuclear maturation rate reached statistical significance in the $1 \mathrm{nM}$ MT group and the $10^{2} \mathrm{nM}$ MT group. Similar situation could also be observed at $48 \mathrm{~h}$ of culture.

Low concentration of MT could also increase nuclear maturation rate of the MI oocytes. As early as $12 \mathrm{~h}$ of culture, nuclear maturation rate of the low-MT groups was higher than the control group. Notably, $1 \mathrm{nM}$ MT was the optimal concentration for MI oocytes, that its nuclear maturation rate was statistically higher than that of any other groups at $18 \mathrm{~h}$ and $24 \mathrm{~h}$.
Fig. 2 Effects of different concentrations of MT on nuclear maturity of GV oocytes and MI oocytes of human. a Effects of different concentrations of MT on GV oocytes. b Effects of different concentrations of MT on MI oocytes. Dotted line = significant increase of nuclear maturity rate between the two adjacent observing time points; Square frame $=$ significant difference compared to the control group; Rectangular frame $=$ significant difference compared to the controls and significant difference between the value of the upper frame and that of the lower frame; MT = melatonin

\section{a}

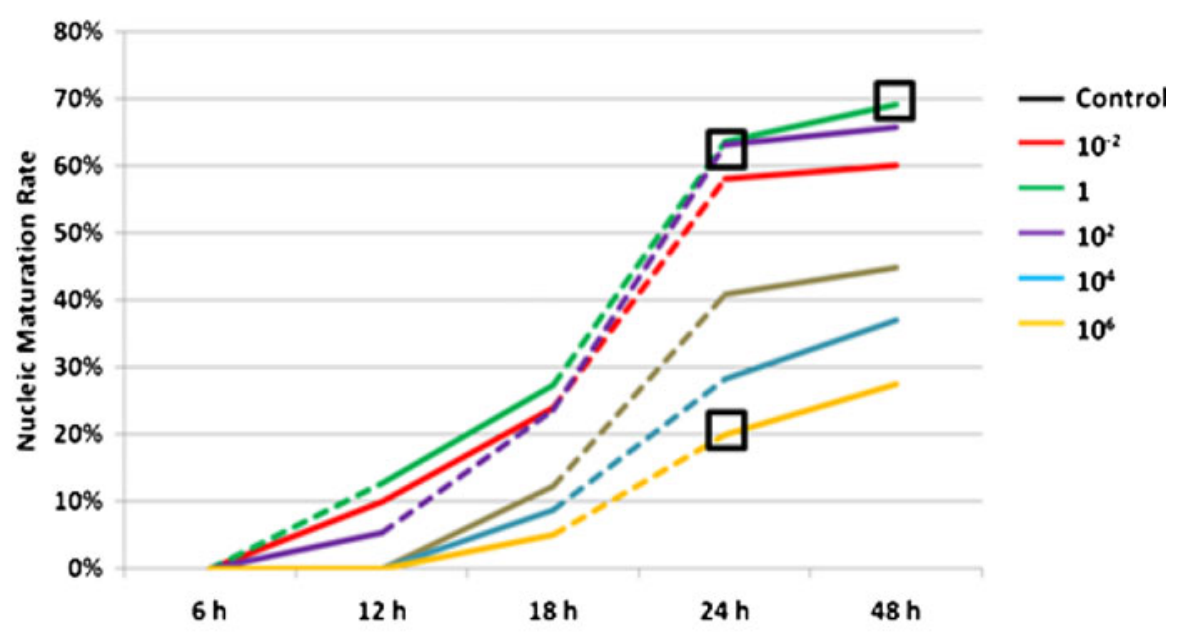

b

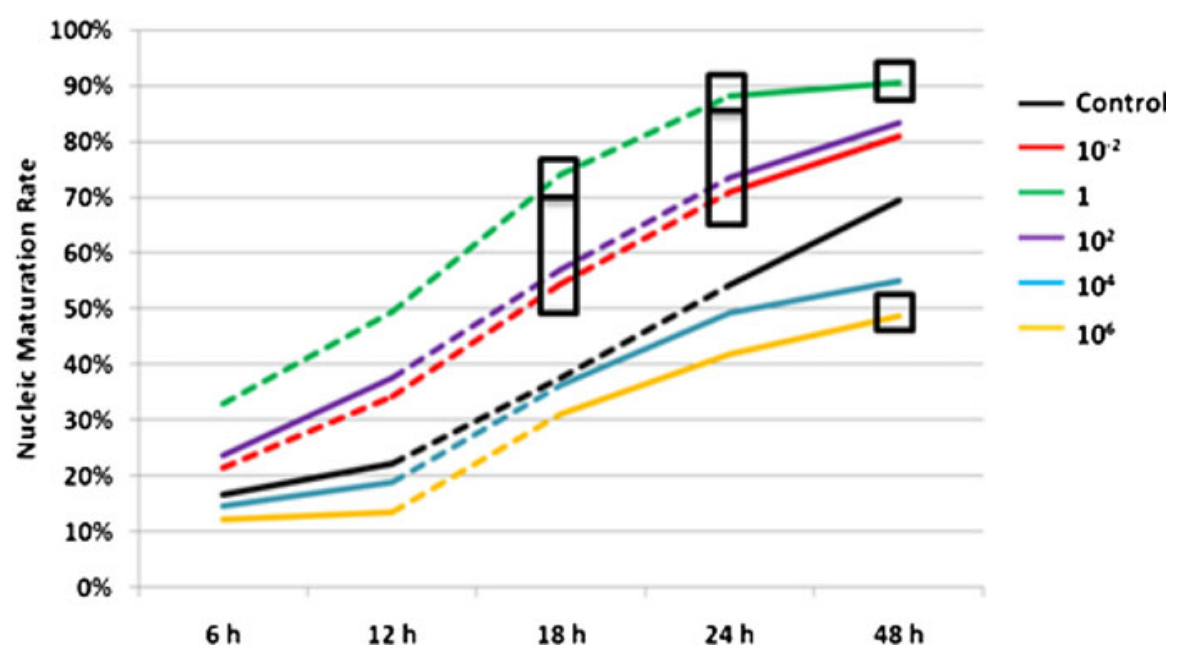


High concentration of MT decreased nuclear maturation rate

For both the GV and MI oocytes, nuclear maturation rate of the high-MT groups was lower than that of the controls. And nuclear maturation rate of GV oocytes and MI oocytes cultured in $10^{6}$ MT media at $24 \mathrm{~h}$ and $48 \mathrm{~h}$ was significantly lower than that of the controls respectively.

\section{Discussion}

Our study demonstrates that low concentrations of MT could improve nuclear maturation of human oocytes during rescue IVM. Low concentrations $\left(10^{-2} \mathrm{nM}, 1 \mathrm{nM}\right.$ and $10^{2} \mathrm{nM}$ ) of MT accelerated meiosis and raised the nuclear maturation rate of human oocytes after vitrification. The best maturation rate of both GV and MI oocytes had been achieved when MT concentration was $1 \mathrm{nM}$.

Previous studies indicated that in IVM cycles, the oocytes matured early (in the first $24 \mathrm{~h}$ ) had significantly higher fertilization rate and cleavage rate than those that matured late [24]. Other studies showed that oocytes matured early yielded significantly higher fertilization and developmental potential than their counterparts matured late $[1,23]$. In the current study, meiotic maturation was evaluated at different time points $(6 \mathrm{~h}, 12 \mathrm{~h}, 18 \mathrm{~h}, 24 \mathrm{~h}$ and $48 \mathrm{~h})$, so that we can identify the optimal culture duration. We found that, regardless of MT concentrations, the nuclear maturation rate at $24 \mathrm{~h}$ was significantly higher than that at $18 \mathrm{~h}$. And if the culture was extended to $48 \mathrm{~h}$, there was no significant raise found in maturation rate. Prolonged culture may impair developmental potential of the oocytes. Therefore, $24 \mathrm{~h}$ is the optimal culture duration to achieve nuclear maturation in our rescue IVM system.

According the result showed in Fig. 2, at $24 \mathrm{~h}$, the highest nuclear maturation rate was found in the $1 \mathrm{nM}$ MT group, though the difference was not significant in GV oocytes. This may have resulted from either a relatively lower number of GV oocytes in our study or due to the inherent differences in the maturational process as compared to M1 oocytes. Thus, more GV oocytes are needed in further studies. However, for oocytes at GV and MI stage, supplementing high concentrations of MT $\left(10^{5} \mathrm{nM}\right.$ and $\left.10^{7} \mathrm{nM}\right)$ resulted in lower maturation rate compared to the controls. And among GV oocytes, the difference between the $10^{7} \mathrm{nM}$ MT group and controls was significant $(P<0.05)$. Therefore, high concentrations of MT $\left(10^{5} \mathrm{nM}\right.$ and $10^{7} \mathrm{nM}$ ) hampered nuclear maturation. Increased MT in follicular fluid seems to have an important role in ovulation [22], but according to the current study overdose MT maybe have detrimental effects on oocyte maturation. Previous studies indicated that physiological concentrations of ROS were necessary for meiotic resumption [25]. And in the current study, supplementing low concentrations of MT balance the extra
ROS which is caused by in vitro manipulation, while supplementing high concentration of MT depleted all the ROS, which resulted in low nuclear maturation rate.

In most of the superovulation cycles, a small portion of oocytes remains immature after hCG trigger. These oocytes are routinely abandoned because clinical outcomes of these oocytes are unsatisfactory [26]. This type of oocyte was utilized in the current study because this is the very first application of MT on human oocytes. Additionally, all these oocytes were thawed after vitrification. Moreover, in order to judge meiotic status as soon as possible, the oocytes were from ICSI cycles and denuded, whereas the oocyte cumulus complexes (OCC) are recommended to be kept intact in traditional IVM manipulation. Both the source of oocytes and vitrification may have detrimental effects on the oocytes in our study, and hence resulted in bias.

In conclusion, our study indicates MT could ameliorate immature human oocyte meiotic maturation. And the maturation rate was the highest when MT concentration was $1 \mathrm{nM} .24 \mathrm{~h}$ are the optimal culture duration to achieve nuclear maturation in our rescue IVM system. However the current study is flawed mainly because of the suboptimal source of the oocytes. MT, as a promising IVM supplement, deserved further studied in the future. Oocytes from conventional IVM cycles are preferable and fertilization, embryogenesis and implantation should be recorded in future studies.

\section{References}

1. Balakier H, Sojecki A, Motamedi G, Librach C. Timedependent capability of human oocytes for activation and pronuclear formation during metaphase II arrest. Hum Reprod. 2004;19(4):982-7.

2. Chan W, Ng T. Development of pre-implantation, mouse embryos under the influence of pineal indoles. J Neural Transm Gen Sect JNT. 1994;96(1):19-29.

3. Chian R-C, Lim J-H, Tan S-L. State of the art in in-vitro oocyte maturation. Curr Opin Obstet Gynecol. 2004;16(3):211.

4. Combelles CM, Gupta S, Agarwal A. Could oxidative stress influence the in-vitro maturation of oocytes? Reprod BioMed Online. 2009; 18(6):864-80.

5. Edwards RG. Maturation in vitro of human ovarian oöcytes. Lancet. $1965 ; 2(7419): 926$.

6. Gardner DK. Textbook of assisted reproductive techniques: laboratory and clinical perspectives. Taylor \& Francis; 2001.

7. Guerin P, El Mouatassim S, Menezo Y. Oxidative stress and protection against reactive oxygen species in the pre-implantation embryo and its surroundings. Hum Reprod Updat. 2001;7(2):175-89.

8. Hardeland R. Atioxidative protection by melatonin. Endocrine. 2005;27(2):119-30.

9. Harvey AJ, Kind KL, Thompson JG. REDOX regulation of early embryo development. Reproduction. 2002;123(4):479-86.

10. Ishizuka B, Kuribayashi Y, Murai K, Amemiya A, Itoh MT. The effect of melatonin on in vitro fertilization and embryo development in mice. J Pineal Res. 2000;28(1):48-51. 
11. Jahnke G, Marr M, Myers C, Wilson R, Travlos G, Price C. Maternal and developmental toxicity evaluation of melatonin administered orally to pregnant Sprague-Dawley rats. Toxicol Sci. 1999;50(2):271-9.

12. Legge M, Sellens MH. Free radical scavengers ameliorate the 2-cell block in mouse embryo culture. Hum Reprod. 1991;6(6):867-71.

13. Leon J, Acuña-Castroviejo D, Escames G, Tan DX, Reiter RJ. Melatonin mitigates mitochondrial malfunction. J Pineal Res. 2005;38(1):1-9.

14. McElhinny AS, Davis FC, Warner CM. The effect of melatonin on cleavage rate of $\mathrm{C} 57 \mathrm{BL} / 6$ and $\mathrm{CB} \mathrm{A} / \mathrm{Ca}$ preimplantation embryos cultured in vitro. J Pineal Res. 1996;21(1):44-8.

15. Nogueira D, Sadeu JC, Montagut J. In vitro oocyte maturation: current status. In: Seminars in reproductive medicine. Thieme Medical Publishers; 2012. p. 199-213.

16. Pang S, Li L, Ayre E, Pang C, Lee P, Xu R, et al. Neuroendocrinology of melatonin in reproduction: recent developments. J Chem Neuroanat. 1998;14(3):157-66.

17. Papis K, Poleszczuk O, Wenta-Muchalska E, Modlinski JA. Melatonin effect on bovine embryo development in vitro in relation to oxygen concentration. J Pineal Res. 2007;43(4):321-6.

18. Rao GD, Chian R, Son W, Gilbert L, Tan S. Fertility preservation in women undergoing cancer treatment. Lancet. 2004;363(9423):1829-9.

19. Reiter RJ. Melatonin and human reproduction. Ann Med. 1998;30(1):103-8.

20. Reiter RJ. Melatonin: clinical relevance. Best Pract Res Clin Endocrinol Metab. 2003;17(2):273-85.

21. Reiter RJ, Tan D-X, Osuna C, Gitto E. Actions of melatonin in the reduction of oxidative stress. J Biomed Sci. 2000;7(6):444-58.

22. Shu Y, Gebhardt J, Watt J, Lyon J, Dasig D, Behr B. Fertilization, embryo development, and clinical outcome of immature oocytes from stimulated intracytoplasmic sperm injection cycles. Fertil Steril. 2007;87(5):1022-7.
23. Son W-Y, Chung J-T, Demirtas E, Holzer H, Sylvestre C, Buckett $\mathrm{W}$, et al. Comparison of in-vitro maturation cycles with and without in-vivo matured oocytes retrieved. Reprod BioMed Online. 2008;17(1):59-67.

24. Son W-Y, Lee S-Y, Lim J-H. Fertilization, cleavage and blastocyst development according to the maturation timing of oocytes in in vitro maturation cycles. Hum Reprod. 2005;20(11):3204-7.

25. Takami M, Preston S, Toyloy V, Behrman HR. Antioxidants reversibly inhibit the spontaneous resumption of meiosis. Am J Physiol Endocrinol Metab. 1999;276(4):E684-8.

26. Tamura H, Takasaki A, Taketani T, Tanabe M, Kizuka F, Lee L, et al. The role of melatonin as an antioxidant in the follicle. J Ovarian Res. 2012;5(5):5.

27. Tan D-X, Manchester LC, Reiter RJ, Plummer BF, Limson J, Weintraub ST, et al. Melatonin directly scavenges hydrogen peroxide: a potentially new metabolic pathway of melatonin biotransformation. Free Radic Biol Med. 2000;29(11):117785.

28. Tan D, Reiter RJ, Manchester LC, Yan M, El-Sawi M, Sainz RM, et al. Chemical and physical properties and potential mechanisms: melatonin as a broad spectrum antioxidant and free radical scavenger. Curr Top Med Chem. 2002;2(2):181-97.

29. Tomás-Zapico C, Coto-Montes A. A proposed mechanism to explain the stimulatory effect of melatonin on antioxidative enzymes. J Pineal Res. 2005;39(2):99-104.

30. Veeck L, Wortham Jr J, Witmyer J, Sandow B, Acosta A, Garcia J, et al. Maturation and fertilization of morphologically immature human oocytes in a program of in vitro fertilization. Fertil Steril. 1983;39(5):594.

31. Wei D, Zhang C, Yin B, Wang P, Xie J, Song X, et al. Early cumulus cell removal could reduce the available embryo rate in human IVF. J Assist Reprod Genet. 2011;28(12):1213-6. 